\title{
Latent Identity Variables: Biometric Matching Without Explicit Identity Estimation
}

\author{
Simon J.D. Prince ${ }^{1}$, Jania Aghajanian ${ }^{1}$, \\ Umar Mohammed $^{1}$, and Maneesh Sahani ${ }^{2}$ \\ ${ }^{1}$ Computer Science \\ ${ }^{2}$ Gatsby Unit, University College London, UK \\ \{s.prince, j.aghajanian, u.mohammed\}@cs.ucl.ac.uk, maneesh@gatsby .ucl.ac.uk \\ http://www.cs.ucl.ac.uk/staff/s.prince
}

\begin{abstract}
We present a new approach to biometrics that makes probabilistic inferences about matching without ever estimating an identity "template". The biometric data is considered to have been created by a noisy generative process. This process consists of (i) a deterministic component, which depends entirely on an underlying representation of identity and (ii) a stochastic component which accounts for the fact that two biometric samples from the same person are not identical. In recognition, we make inferences about whether the underlying identity representation is the same without ever estimating it. Instead we treat identity as fundamentally uncertain and consider all possible values in our decision. We demonstrate these ideas with toy examples from face recognition. We compare our approach to the class-conditional viewpoint.
\end{abstract}

Keywords: Biometrics, Face Recognition, Bayesian Methods.

\section{Introduction}

Typical biometric systems have the following structure 3] : features are extracted from a biometric sample to form a biometric template. This represents the discriminative aspects of the sample such as face texture, fingerprint minutiae or iris pattern. In order to compare two templates, a score is calculated representing the similarity of the two templates. This is often based on a distance metric in feature space (e.g.[10]). In face identification we search for the gallery image with maximum similarity to a probe image. In face verification, we impose a threshold, $\mathrm{T}$ on the similarity: if the two biometric samples are not sufficiently similar we infer that they came from different individuals.

Although this approach has yielded much success, it has several drawbacks. First, most algorithms of this type cannot provide an estimate of certainty on the final decision. In real systems, we might wish to defer decision making and gather more data if we are not confident. Second, there is no principled way to deal with multiple gallery and/or probe observations from the same individual. Third, there is no easy way to incorporate prior information about the relative likelihood of different matching hypotheses. For example, the time of day 
or location might change the likelihood of different individuals being present. Fourth, it is not obvious how to construct non-uniform similarity metrics that address the fact that different regions of the feature space may be differently discriminable. Fifth, it is hard to incorporate the possibility of errors in the preprocessing into the decision metric. For example, in fingerprint recognition, the preprocessing algorithm might misclassify minutiae, or in face recognition the face features might be mis-localized. If this process is not modelled, erroneous matching decisions will result. Finally, these algorithms struggle in the presence of nuisance variables which change the appearance of observations. For example, in face recognition, pose and illumination changes reduce matching performance.

The emphasis in these approaches is on extracting a template which is essentially a representation of identity. In this paper, we pose the following philosophical question: is it necessary to estimate identity to make inferences about whether biometric samples match?

In recent work in face recognition, we have introduced a new framework for biometric matching 9 . We treat the data as having been generated from a smaller number of underlying variables. Some of these variables are pure representations of identity (i.e. signal), whereas others may represent irrelevant extrinsic factors such as capture conditions. The generative process also contains stochastic elements, which explain why the measured biometric data varies, even when the identity of the individual and the capture conditions are identical. In recognition, we pose the question: what is the likelihood that the underlying identity variables that explain two biometric samples were the same? We estimate this likelihood regardless of the actual values of these variables and the other contributing factors. This approach has proven extremely successful when applied to face recognition across pose 9 .

The goal of this paper is to introduce this approach to the wider biometric community. In Sections 2 and 3 we present a simple tutorial example in the domain of face recognition and give examples of both identification and verification. In Section 4 we discuss more sophisticated examples of this approach. In Section [5] we discuss how the advantageous features of our approach might be applied to other biometrics. Finally, in Section 6 we describe how our approach can be rationalized with a class-conditional viewpoint.

\section{Latent Identity Variable Models}

Underpinning our approach is the attempt to explain variation in biometric data with a structured model. Imagine that we have $\mathrm{J}$ measurements from each of I individuals in a training database. We denote the j'th measurement of the i'th individual as $\mathbf{x}_{i j}$. In the simplest case we will consider models of the form

$$
\mathbf{x}_{i j}=g\left(\mathbf{h}_{i}, \theta\right)+\epsilon_{i j} .
$$

The term $\mathbf{h}_{i}$ is known as a latent identity variable (LIV). This is a pure representation of identity which contains no noise or nuisance factors and hence only depends on the individual $\mathrm{i}$, not the instance, $\mathrm{j}$. The data $\mathbf{x}_{i j}$ are modelled as 
being generated by the operation of some function $\mathrm{g}($.$) on the LIV plus additive$ Gaussian noise $\epsilon_{i j}$ with diagonal covariance $\Sigma$. The function $g($.$) depends on$ some fixed parameters $\theta$.

The two terms of the model divide the data into signal and noise components. The first (signal) term is deterministic in that it depends only on the identity of the individual. The second (noise) term is stochastic and explains why each measurement of the same individual differs. The term 'latent' (or equivalently 'hidden') indicates that although the identity variable $\mathbf{h}_{i}$ explains some of the observed data variation, it cannot be directly measured: since generation is noisy, we can never know exactly what value it takes for a given observation $\mathbf{x}_{i j}$. The best that we can do is to make probabilistic statements about its likely values.

\section{Single Feature Model}

In order to make these ideas concrete, we will provide a very simple example. We will consider the generative model:

$$
x_{i j}=\mu+f h_{i}+\epsilon_{i j}
$$

where $x_{i j}$ is a single scalar biometric measurement (e.g. height) from the j'th measurement of the i'th training individual. This is explained by a signal term $\mu+f h_{i}$ which is a function $g($.$) of the latent identity variable h_{i}$ and a noise term $\epsilon_{i j}$ which is Gaussian with mean 0 and with variance $\sigma^{2}$. We can alternatively write the model in terms of conditional probability:

$$
\begin{aligned}
\operatorname{Pr}\left(x_{i j} \mid h_{i}, \mu, f, \Sigma\right) & =\mathcal{G}_{x_{i j}}\left[\mu+f h_{i}, \sigma^{2}\right] \\
\operatorname{Pr}\left(h_{i}\right) & =\mathcal{G}_{h_{i}}[0,1] .
\end{aligned}
$$

where $\mathcal{G}_{a}[b, c]$ represents a Gaussian distribution in $a$, with mean $b$ and (co)variance $c$. In order to complete the model, we have also defined a prior distribution over the latent identity variable, $h_{i}$. This is defined to be Gaussian with zero mean and unity variance. There are two phases to using the LIV model:

- Learning: in the learning phase, we learn the parameters $\mu, f, \sigma$ from a training set of data $x_{1 \ldots I, 1 \ldots J}$.

- Recognition: in the recognition phase, we use this model to infer whether test data originates from the same person.

\subsection{Learning}

In learning, we want to find the values of the parameters $\Theta=\{\mu, f, \sigma\}$ under which the training data is most likely. This would be easy if we knew the identity variables $h_{i}$, but unfortunately these are unknown as well. Likewise, it would be easy to estimate the identity variables $h_{i}$ if we already knew the parameters $\Theta=\{\mu, f, \sigma\}$. Fortunately, there is a well-established solution to this "chicken and egg" problem: we use the Expectation Maximization (EM) algorithm [4 to alternately estimate the two sets of parameters in such a way that guarantees the likelihood of the data will increase at each stage (see Appendix A). 


\subsection{Recognition}

In recognition we do not know the relationship between different individuals I and the data vectors. We denote the k'th hypothesized relationship a "model", $\mathcal{M}_{k}$. We can calculate the likelihood of the data given a model $\mathcal{M}$ using:

$$
\operatorname{Pr}(X \mid \Theta, \mathcal{M})=\prod_{i=1}^{I} \int \prod_{j=1}^{N_{I}} \operatorname{Pr}\left(x_{i j}, h_{i} \mid \Theta\right) d \mathbf{h}_{i}
$$

In this expression we calculate the total likelihood of all of the data $X$ for model $\mathcal{M}$ by (i) writing the joint likelihood of the observed and hidden variables and (ii) marginalizing (integrating out) the unknown hidden variables. Finally, we compare these models using Bayes' rule:

$$
\operatorname{Pr}\left(\mathcal{M}_{k} \mid X\right)=\frac{\operatorname{Pr}\left(\mathbf{x} \mid \mathcal{M}_{k}\right) \operatorname{Pr}\left(\mathcal{M}_{k}\right)}{\sum_{l=0}^{N} \operatorname{Pr}\left(\mathbf{x} \mid \mathcal{M}_{l}\right) \operatorname{Pr}\left(\mathcal{M}_{l}\right)}
$$

We include priors $\operatorname{Pr}\left(\mathcal{M}_{k}\right)$ which allow us to incorporate our prior expectations as to which measurements belong together. To illustrate these ideas we will give two concrete examples: Identification and Verification.

\section{(A) CLOSED SET IDENTIFICATION}

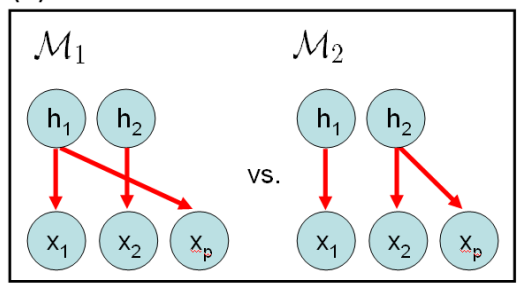

\section{(B) VERIFICATION}

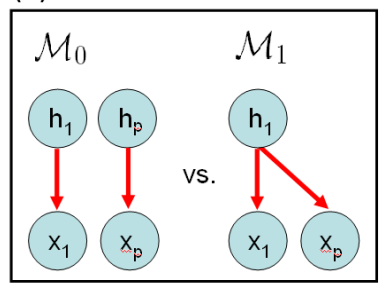

Fig. 1. Relationship between latent identity variables (top) and measurements (bottom) for identification (A) and verification tasks (B). Recognition is framed as comparing models $\mathcal{M}_{k}$ of the data. Each model represents a different relationship between the observed measurements $\mathrm{x}$ and the latent identity variables $\mathrm{h}$. If two measurements come from the same individual then the underlying identity variable is the same.

(A) Identification: Consider identification with two data points in the gallery $x_{1}, x_{2}$ and a probe data point $x_{p}$. In model $\mathcal{M}_{1}$ the probe data matches the first gallery data, and in model $\mathcal{M}_{2}$, the probe matches the second gallery data. The first gallery data $x_{1}$ and the probe data $x_{p}$ are both explained by identity variable $h_{1}$. The second gallery data has its own latent identity variable $h_{2}$. Conversely, in model $\mathcal{M}_{2}$, the probe face matches the second gallery face and so these share an identity variable $h_{2}$ (see Figure[A). We write the likelihood of the first model as:

$$
\begin{aligned}
\operatorname{Pr}\left(x_{1,2, p} \mid \mathcal{M}_{1}\right) & =\int \operatorname{Pr}\left(x_{1}, x_{p}, h_{1}\right) d h_{1} \int \operatorname{Pr}\left(x_{2}, h_{2}\right) d h_{2} \\
& =\int \operatorname{Pr}\left(x_{1} \mid h_{1}\right) \operatorname{Pr}\left(x_{p} \mid h_{1}\right) \operatorname{Pr}\left(h_{1}\right) d h_{1} \int \operatorname{Pr}\left(x_{2} \mid h_{2}\right) \operatorname{Pr}\left(h_{2}\right) d h_{2}
\end{aligned}
$$


where in the second line, we have written the joint probabilities as conditional probabilities. Notice that all of the terms in the second line were defined in Equations 3 and 4 Similarly, the likelihood for model $\mathcal{M}_{2}$ can be written:

$$
\operatorname{Pr}\left(x_{1,2, p} \mid \mathcal{M}_{2}\right)=\int \operatorname{Pr}\left(x_{1} \mid h_{1}\right) \operatorname{Pr}\left(h_{1}\right) d h_{1} \int \operatorname{Pr}\left(x_{2} \mid h_{2}\right) \operatorname{Pr}\left(x_{p} \mid h_{2}\right) \operatorname{Pr}\left(h_{2}\right) d h_{2}
$$

(B) Verification: In verification we have two biometric data measurements $x_{1}$ and $x_{p}$, and we would like to say whether these belong to different people (model $\mathcal{M}_{0}$ ) or the same person (model $\mathcal{M}_{1}$ ). In model $\mathcal{M}_{0}$ the measurements $x_{1}$ and $x_{p}$ do not match, so each has its own identity variable $\left(h_{1}\right.$ and $\left.h_{p}\right)$. In model $\mathcal{M}_{1}$ the measurements match and are both explained by one identity variable $h_{1}$ (see Figure 1B). The likelihoods can be written:

$$
\begin{aligned}
& \operatorname{Pr}\left(x_{1, p} \mid \mathcal{M}_{0}\right)=\int \operatorname{Pr}\left(x_{1} \mid h_{1}\right) \operatorname{Pr}\left(h_{1}\right) d h_{1} \int \operatorname{Pr}\left(x_{p} \mid h_{p}\right) \operatorname{Pr}\left(h_{p}\right) d h_{p} \\
& \operatorname{Pr}\left(x_{1, p} \mid \mathcal{M}_{1}\right)=\int \operatorname{Pr}\left(x_{1} \mid h_{1}\right) \operatorname{Pr}\left(x_{p} \mid h_{1}\right) \operatorname{Pr}\left(h_{1}\right) d h_{1}
\end{aligned}
$$

One might be concerned about this comparison: there are more underlying identity variables $\mathrm{h}$ in model $\mathcal{M}_{0}$ than there are in model $\mathcal{M}_{1}$, so surely the data is bound to be more likely under this model? In fact this is not true since we have integrated these variables out of the final solution. This is known as Bayesian model selection and is a valid way of comparing models of different dimensionality [7. For the model described in Equation 2, it can be shown that:

$$
\int \operatorname{Pr}\left(x_{1 \ldots N}, h\right)=N \sigma^{2} f^{-2}(2 \pi)^{-D_{h} / 2} \prod_{i=1}^{N} \mathcal{G}_{\hat{x}_{i}}\left[0, \sigma^{2}\right] / \mathcal{G}_{y}\left[0, N \sigma^{-2} f^{2}+1\right]
$$

where $y=f \sigma^{-2} \sum \hat{x}_{i}$ and $\hat{x}_{i}=x_{i}-\mu$. Note that we don't estimate the underlying identity representation. Instead we ask: were two biometric samples generated from the same underlying identity, regardless of what that identity was?

\subsection{Face Recognition Example: Independent Pixel Model}

To validate these ideas, we present a face recognition example using the XM2VTS database 12. We spatially register each RGB image, resize to $70 \times 70$ pixels and vectorise the pixel contents, to form training data vectors $\mathbf{x}_{i j}$, each of dimension $d=70 \times 70 \times 3=14700$. We describe the generation process as:

$$
\mathbf{x}_{i j}=\mu+\mathbf{F} \mathbf{h}_{i}+\epsilon_{i j}
$$

where $\mathbf{F}$ is a diagonal $d \times d$ matrix, $\mu$ is a vector representing the mean face and $\epsilon_{i j}$ is a multivariate Gaussian variable representing noise with diagonal 


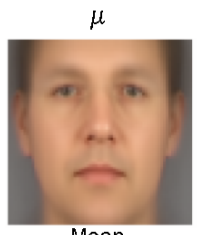

Mean

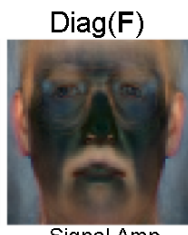

Signal Amp.

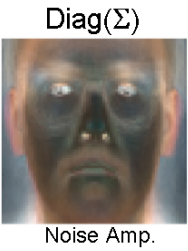

Noise Amp.
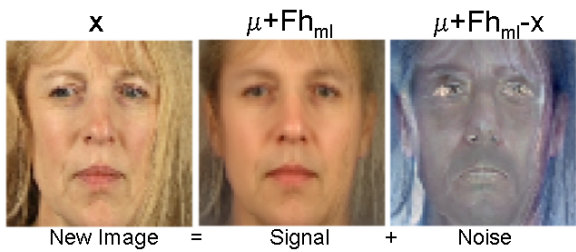

Fig. 2. Results from the "Independent Pixels" face recognition model. The first three panels indicate the results of the learning phase, $\Theta=\{\mu, f, s\}$. The fourth panel is a new face, which has been decomposed into the signal (panel 5) and noise (panel 6) components. Note that this is only the most likely decomposition. In our recognition metric, all possible decompositions are taken into account.

covariance $\Sigma$. As before, we assume a Gaussian prior over the latent identity variable $\mathbf{h}_{i}$ with zero mean and identity covariance. This model is a multivariate version of Equation 2 where each pixel is treated as independent. We train this model with 4 examples each of 160 people, using 10 iterations of the EM algorithm. We then performed face identification and verification experiments with 100 pairs of images that were not part of the training data.

In Figure 2 we show the results of the learning process, and for a new image, the most likely apportioning of signal and noise. For this toy model, recognition performance was $60 \%$. In the next section, we present a series of increasingly sophisticated LIV models. In Section [5] we discuss other aspects of generative models which might be applied to biometric data.

\section{A Family of Abstract LIV Models}

In Figure 3 we present a series of more sophisticated abstract Latent Identity Variable models. We use the term abstract to denote the fact that they do not encompass any information that is specific to the biometric domain. The "Factor Analyzer" model permits linear correlations between the measurements, but maintains an independent noise model. In face recognition, this is similar to the eigenfaces approach[10. The "Probabilistic Linear Discriminant Analyzer" model also models correlations in the noise. Here, there is a second latent variable $\nu_{i j}$, which is not associated with identity, which must also be integrated out of the final formulation.

The "Mixture of Gaussians" model is particularly interesting in that the representation of the signal is entirely discrete: the signal is described as being one of $\mathrm{L}$ canonical data points $\mu_{l}$. Here, the identity vector $l_{i}$ simply describes which of these canonical examples we are seeing. An example of this type of model is explored for the case of face recognition in Figure 4A. Combining the previously discussed models leads naturally to the "Mixture of Factor Analyzers" and 


\begin{tabular}{|c|c|c|c|}
\hline Model Specification & Data & Signal & Noise \\
\hline $\begin{array}{c}\mathbf{x}_{i j}=\boldsymbol{\mu}+\mathbf{F} \mathbf{h}_{i}+\boldsymbol{\varepsilon}_{i j} \\
\operatorname{Pr}\left(\mathbf{h}_{i}\right)=\mathscr{G}_{\mathbf{h}}[\mathbf{0}, \mathbf{I}] \operatorname{Pr}\left(\epsilon_{i j}\right)=\mathcal{G}_{\epsilon}[\mathbf{0}, \boldsymbol{\Sigma}] \\
\mathbf{F} \Sigma \text { diagonal } \\
\text { Independent Pixel Model }\end{array}$ & 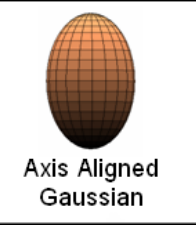 & $\begin{array}{l}\text { Axis Aligned } \\
\text { Gaussian }\end{array}$ & Axis Aligned \\
\hline $\begin{array}{c}\mathbf{x}_{i j}=\boldsymbol{\mu}+\mathbf{F} \mathbf{h}_{i}+\boldsymbol{\varepsilon}_{i j} \\
\operatorname{Pr}\left(\mathbf{h}_{i}\right)=\mathscr{G}_{\mathbf{h}}[\mathbf{0}, \mathbf{I}] \quad \operatorname{Pr}\left(\epsilon_{i j}\right)=\mathcal{G}_{\epsilon}[\mathbf{0}, \mathbf{\Sigma}] \\
\Sigma \text { diagonal } \\
\text { Factor Analyzer }\end{array}$ & Factor Analyzer & Subspace & $\begin{array}{c}\text { Axis Aligned } \\
\text { Gaussian (uniform) }\end{array}$ \\
\hline $\begin{array}{c}\mathbf{x}_{i j}=\mu+\mathbf{F h} \mathbf{h}_{i}+\mathbf{G} v_{i j}+\varepsilon_{i j} \\
\operatorname{Pr}\left(\mathbf{h}_{i}\right)=\mathscr{G}_{\mathbf{h}}[\mathbf{0}, \mathbf{I}] \operatorname{Pr}\left(\epsilon_{i j}\right)=\mathcal{G}_{\epsilon}[\mathbf{0}, \mathbf{\Sigma}] \\
\operatorname{Pr}\left(v_{i j}\right)=\mathscr{G}_{v}[\mathbf{0}, \mathbf{I}] \quad \Sigma \text { diagonal } \\
\text { Probabilistic LDA }\end{array}$ & Factor Analyzer & & $\begin{array}{l}\begin{array}{c}\text { Factor Analyzer } \\
\text { (uniform) }\end{array} \\
\end{array}$ \\
\hline $\begin{array}{c}\mathbf{x}_{i j}=\mu_{l_{i}}+\mathcal{E}_{i j} \\
\operatorname{Pr}\left(\epsilon_{i j}\right)=\mathcal{G}_{\epsilon}\left[0, \Sigma_{l_{i}}\right] \operatorname{Pr}\left(l_{i}=a\right)=f_{a} \\
\Sigma_{l_{i}} \text { diagonal } \\
\text { Mixture of Gaussians }\end{array}$ & $\begin{array}{l}\text { Mixture of } \\
\text { Gaussians }\end{array}$ & Discrete Points & $\begin{array}{c}\text { Axis aligned } \\
\text { Gaussian }\end{array}$ \\
\hline $\begin{array}{c}\mathbf{x}_{i j}=\mu_{l_{i}}+\mathbf{F}_{l_{i}} \mathbf{h}_{i}+\varepsilon_{i j} \\
\operatorname{Pr}\left(\mathbf{h}_{i}\right)=\mathscr{G}_{\mathbf{h}}[\mathbf{0}, \mathbf{I}] \operatorname{Pr}\left(\epsilon_{i j}\right)=\mathcal{G}_{\epsilon}\left[0, \Sigma_{l_{i}}\right] \\
\operatorname{Pr}\left(l_{i}=a\right)=f_{a} \quad \Sigma_{l_{i}} \text { diagonal } \\
\text { Mixture of Factor Analyzers }\end{array}$ & $\begin{array}{l}\text { Mixture of Factor } \\
\text { Analyzers }\end{array}$ & $\begin{array}{l}\text { Mixture of } \\
\text { Subspaces }\end{array}$ & $\begin{array}{c}0 \\
\text { Axis aligned } \\
\text { Gaussian }\end{array}$ \\
\hline $\begin{array}{c}\mathbf{x}_{i j}=\mu_{l_{i}}+\mathbf{F}_{l_{i}} \mathbf{h}_{i}+\mathbf{G}_{l_{i}} v_{i}+\varepsilon_{i j} \\
\operatorname{Pr}\left(\mathbf{h}_{i}\right)=\mathscr{G}_{\mathbf{h}}[\mathbf{0}, \mathbf{I}] \quad \operatorname{Pr}\left(\epsilon_{i j}\right)=\mathcal{G}_{\epsilon}\left[\mathbf{0}, \Sigma_{l_{i}}\right] \\
\operatorname{Pr}\left(l_{i}=a\right)=f_{a} \quad \Sigma_{l_{i}} \text { diagonal } \\
\quad \operatorname{Pr}\left(v_{i j}\right)=\mathscr{G}_{v}[\mathbf{0}, \mathbf{I}] \\
\text { Mixture of Probabilistic LDAs }\end{array}$ & $\begin{array}{l}\text { Mixture of Factor } \\
\text { Analyzers }\end{array}$ & $\begin{array}{l}\text { Mixture of } \\
\text { Subspaces }\end{array}$ & $\begin{array}{ccc}0 & 0 \\
0 & 0 & 0 \\
\text { Factor Analyzer } & 0\end{array}$ \\
\hline
\end{tabular}

Fig. 3. Examples from the family of linear additive Latent Identity Variable models. Each row represents a different model, as defined by the first column. The second column depicts the probability model for the data as a whole. This is composed of the signal component (third column), which provides information about identity and the noise component (fourth column) which does not.

"Mixture of Probabilistic LDAs" models. The latter can potentially describe an arbitrary signal distribution, combined with an arbitrary noise distribution that varies with the position in data space. 


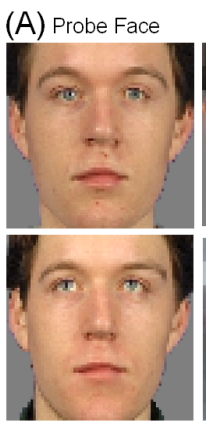

Gallery Face

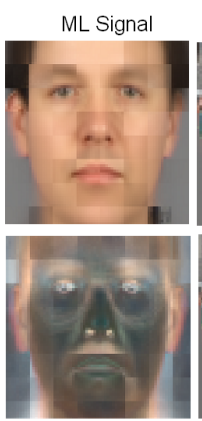

Noise Amp
ML Noise
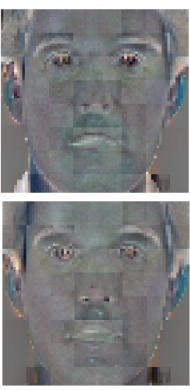

ML Noise
(B)

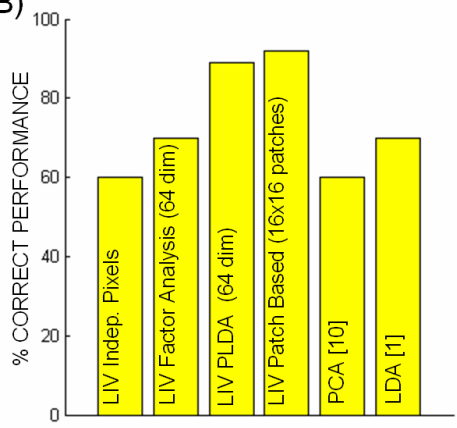

Fig. 4. (A) Results of "Mixture of Gaussians" model. Faces are represented as a regular grid of patches. Each patch is taken from a library with a finite discrete set of 16 possibilities. Hence the identity variable holds a set of indices to the library. The topcenter panel shows the approximation that explains the probe and gallery images best (signal). The right panel shows the residual noise component. (B) Empirical comparison for several LIV models and two well-known methods for face identification with 100 XM2VTS images. Task is difficult as (i) training and gallery sets are not overlapping (ii) there is no attempt to compensate for photometric variation (iii) registration is poor (iv) images were captured in different recording sessions.

\section{Strengths of Generative Modelling Approach}

Domain Specific Information: up until now, all of the models used have been very general. However, it is quite possible to incorporate domain specific information about how the measurements were generated. For example, in face recognition, we might explicitly describe the lighting and 3D projection process as in 2]. Similarly, in fingerprint analysis, we could model the smudging and printing process, and in iris recognition we might model image blurring.

Missing Data: generative models can handle missing data, by including a binary latent variable encoding the presence or absence of a feature. This could be used in fingerprint recognition where minutiae may be missed or misclassified.

Invariance to transformations: many biometrics require spatial registration of data before comparison. This registration is prone to error. By treating the transformation parameters as hidden variables (i.e. as fundamentally uncertain), we can make the system invariant to these transformations and robust in cases where the transformation parameters are ambiguous.

Multiple Appearances: generative models can also describe situations in which the same underlying identity created quite different looking observations [9]. For example, fingerprint data differ depending on the type of scanner used to collect it, and face images may differ depending on the expression.

In all the models from Section 4, the integral in the recognition step can be calculated exactly. However, this may not be true for complex domain specific 
models. In such cases, we can approximate the posterior distribution for $\mathbf{h}$ using variational inference [6], sampling methods or Laplace's approximation[7].

\section{Relationship to Class Conditional Viewpoint}

In this section, we discuss how the LIV method relates to a more conventional class-conditional viewpoint. Here, each gallery data point induces a density $\operatorname{Pr}\left(x_{p} \mid x_{i}, \mathcal{M}_{i}\right)$ around it. We calculate the probability of seeing probe data $x_{p}$ given each gallery data point $x_{i}$. If $\mathcal{M}_{i}$ is the model that groups the probe biometric sample $x_{p}$ with the i'th gallery biometric sample $x_{i}$ then

$$
\begin{aligned}
\operatorname{Pr}\left(X \mid \mathcal{M}_{i}\right) & =\prod_{k \neq i} \operatorname{Pr}\left(x_{k} \mid \Theta, \mathcal{M}_{i}\right) \operatorname{Pr}\left(x_{i}, x_{p} \mid \Theta, \mathcal{M}_{i}\right) \\
& =\prod_{k \neq i} \operatorname{Pr}\left(x_{k} \mid \Theta\right) \operatorname{Pr}\left(x_{i} \mid \Theta, \mathcal{M}_{i}\right) \operatorname{Pr}\left(x_{p} \mid x_{i}, \Theta, \mathcal{M}_{i}\right) \\
& =\prod_{k} \operatorname{Pr}\left(x_{k} \mid \Theta\right) \operatorname{Pr}\left(x_{p} \mid x_{i}, \Theta, \mathcal{M}_{i}\right)
\end{aligned}
$$

The integral over, $h_{i}$ is then buried in the conditional $\operatorname{Pr}\left(x_{p} \mid x_{i}, \Theta, \mathcal{M}_{i}\right)$ :

$$
\begin{aligned}
\operatorname{Pr}\left(x_{p} \mid x_{i}, \Theta, M_{i}\right) & =\int \operatorname{Pr}\left(x_{p} \mid h_{i}, \Theta\right) \operatorname{Pr}\left(h_{i} \mid x_{i}, \Theta\right) d h_{i} \\
& =\frac{1}{\operatorname{Pr}\left(x_{i} \mid \Theta\right)} \int \operatorname{Pr}\left(x_{p} \mid h_{i}, \Theta\right) \operatorname{Pr}\left(x_{i} \mid h_{i}, \Theta\right) \operatorname{Pr}\left(h_{i} \mid \Theta\right) d h_{i}
\end{aligned}
$$

which recovers the form that we originally used. This viewpoint is closely related to Bayesian one shot learning[5]. The training process can be thought of as implicitly learning a prior over face data and combines with the gallery data $x_{i}$ to build a class conditional probability distribution for the gallery individual.

\section{Discussion}

In this paper, we have discussed a new approach to biometric matching. We model observed data as being the result of a generative process, in which some of the component variables are pure representations of identity (latent identity variables). We learn the parameters of this model from training data. In recognition, we calculate the probability that the probe and gallery data were generated from the same underlying identity regardless of what that identity was.

There are several advantages to our formulation. (i) It provides valid posterior probability over possible hypotheses in both verification and identification scenarios. (ii) There is no need to set arbitrary parameters as all components can be learnt. (iii) In our method it is easy to compare biometric data captured in quite different circumstances. (iv) We can easily incorporate prior information about possible data hypotheses. (v) It is easy to incorporate domain specific 
information about the generation process. (vi) There is a clear way to deal with multiple gallery and/or probe data belonging to the same person.

Our method compares favorably to other decision algorithms. Distance based methods (e.g. 10]) cannot always easily be adapted to have these advantages. Decision based on frequentist statistical principles do not provide a method to incorporate prior information. Previous probabilistic approaches (e.g.[11,?]) have been focussed on either identification or verification tasks, but cannot easily handle both.

\section{References}

1. Belhumeur, P.N., Hespanha, J., Kriegman, D.J.: Eigenfaces vs. Fisherfaces: Recognition Using Class Specific Linear Projection. PAMI 19, 711-720 (1997)

2. Blanz, V., Romdhani, S., Vetter, T.: Face identification across different poses and illumination with a 3D morphable model. In: ICFGR, pp. 202-207 (2002)

3. Bolle, R.M., Connell, J.H., Pankathi, S., Ratha, N.K., Senior, A.W.: Guide to Biometrics. Springer, New York (2004)

4. Dempster, A.P., Laird, N.M., Rubin, D.B.: Maximum likelihood for incomplete data via the EM algorithm. Proc. Roy. Stat. Soc. B 39, 1-38 (1977)

5. Fei-Fei, L., Fergus, R., Perona, P.: A Bayesian approach to unsupervised one-shot learning of object categories. In: CVPR, pp. 1134-1141 (2003)

6. Jordan, M.I., Ghahramani, Z., Jaakola, T.S., Saul, L.K.: An introduction to variational methods for graphical models. In: Jordan, M.I. (ed.) Learning in Graphical Models (1998)

7. MacKay, D.: Information theory, inference, and learning algorithms. Cam. U. Press, Cambridge (2003)

8. Moghaddam, B., Jebra, T., Pentland, A.: Bayesian face recognition. Pattern Recognition 3, 1771-1782 (2000)

9. Prince, S.J.D., Elder, J.H.: Tied factor analysis for face recognition across large pose changes. In: BMVC, vol. 3, pp. 889-898 (2006)

10. Turk, M., Pentland, A.P.: Face recognition Using eigenfaces. In: CVPR, pp. 586591 (1991)

11. Zhou, S.K., Chellappa, R.: Probabilistic Identity characterization for face recognition. In: CVPR, vol. 2, pp. 805-812 (2004)

12. http://www.ee.surrey.ac.uk/Research/VSSP/xm2vtsdb/

\section{Appendix A: Learning LIV Models}

In the expectation step (or E-Step) we fix $\Theta$ and find the posterior distribution over the hidden identity variables $h_{i}$. In the maximization step (or M-Step) we re-estimate $\Theta=\{\mu, f, \sigma\}$ given the distribution calculated in the E-Step.

E-Step: In the E-Step, we use Bayes' rule to calculate the posterior distribution of the latent identity variables $h_{i}$ given the data $x_{i j}$ :

$$
\operatorname{Pr}\left(h_{i} \mid x_{i, 1 \ldots J}, \Theta\right)=\frac{\prod_{j=1}^{J} \operatorname{Pr}\left(x_{i j} \mid h_{i}, \Theta\right) \operatorname{Pr}\left(h_{i}\right)}{\int \prod_{j=1}^{J} \operatorname{Pr}\left(x_{i j} \mid h_{i}, \Theta\right) \operatorname{Pr}\left(h_{i}\right) d h_{i}}
$$


where we have assumed that all of the data samples for each person were independent. Notice that all of the terms in this equation were defined in our original description of the generative model in Equations 3 and 4 . For this particular model, substituting in and rearranging gives:

$$
\operatorname{Pr}\left(h_{i} \mid x_{i, 1 \ldots J}, \Theta\right)=\mathcal{G}_{h_{i}}\left[\frac{f}{J f^{2}+\sigma^{2}} \sum_{j=1}^{J}\left(x_{i j}-\mu\right), \frac{\sigma^{2}}{J f^{2}+\sigma^{2}}\right]
$$

M-Step:In the M-Step we update our current estimate of the parameters by optimizing the following expression:

$$
Q\left(\Theta_{t}, \Theta_{t-1}\right)=\sum_{i, j=1}^{I, J} E_{h_{i}}\left[\log \left[\operatorname{Pr}\left(\mathbf{x}_{i j} \mid \mathbf{h}_{i}\right) \operatorname{Pr}\left(\mathbf{h}_{i}\right)\right]\right]
$$

where $t$ is the iteration index and for this model:

$$
E_{h_{i}}\left[\log \left[\operatorname{Pr}\left(\mathbf{x}_{i j} \mid \mathbf{h}_{i}\right) \operatorname{Pr}\left(\mathbf{h}_{i}\right)\right]\right]=\kappa-0.5 \log \left|\sigma^{2}\right|-E_{h_{i}}\left[\left(\frac{x_{i j}-\mu-f h_{i}}{2 \sigma^{2}}\right)^{2}\right]
$$

where $\kappa$ is an unimportant constant. We maximize this with respect to $\mu, f$ and $\sigma$ by taking derivatives and setting the resulting expressions to zero. 